\title{
Effet de la proximité d'un talus sur un pieu court et rigide chargé horizontalement
}

A. BOUAFIA

Institut de Génie civil Université de Blida, Algérie 48, rue Hassiba-Ben-Bouali 16016 Alger, Algérie

\section{A. BOUGUERRA}

Thésard en Génie civil

Université de Rennes 32. rue Mirabeau Bat J 205 35700 Rennes
는 L'influence de la proximité d'une pente sur le comportement d'un pieu court et rigide chargé horizontalement a été étudiée à l'aide d'essais sur modèles réduits centrifugés.

L'étude des déplacements du pieu a montré que l'effet du talus n'est significatif que si le pieu se trouve en deçà d'une distance limite de la tête du talus.

On montre qu'il est possible de prévoir le comportement d'un pieu implanté près d'un talus, en effectuant une correction adéquate des courbes P-Y correspondant à un sol horizontal.

\section{The slope effect on the behaviour of a horizontally loaded short rigid pile}

\footnotetext{
Ul. The influence of the proximity of the slope on the behaviour of a horizontally loaded short rigid pile has been investigated by the reduced scale model tests in centrifuge.

The study of the pile displacements has shown that the effect of the slope is only significant if the pile is closer than a limit distance from the slope top.

In the latest part, it has been shown that it is possible to predict the pile behaviour near a slope by an adequate correction of the P.Y curves of the same pile in a horizontal soil.
} 


\section{NOTATIONS}

a

$\mathrm{C}_{\mathrm{V}}$ : coefficient d'uniformité granulométrique

D : fiche du pieu dans le sol (m)

$\mathrm{d}_{\mathrm{to}}$ : ciamètre efficace des grains ( $\mathrm{mm}$ )

e : cote de l'effort en tête (m)

$\mathrm{E}_{\mathrm{M}} \quad$ : module pressiométrique (MPa)

$E_{p} I_{0}$ : rigidité à la flexion du pieu $\left(\mathrm{MN} \cdot \mathrm{m}^{2}\right)$

$E_{,} \quad$ : module de réaction sécant (MPa)

$\mathrm{E}_{4} \quad$ : module de réaction tangent initial (MPa)

g : accélération de la gravité terrestre $\left(\mathrm{m} . \mathrm{s}^{-2}\right)$

$\mathrm{H} \quad$; effort horizontal en tête du pieu (kN)

ID : indice de densité du sable (en \%)

$I_{E} \quad$ : facteur d'influence du talus sur le module de réaction

$I_{\text {Mmax }}$ : facteur d'influence du talus sur le moment maximal

$I_{Y H}$ : facteur d'influence du talus sur le déplacement en tête

Iy : facteur d'influence du talus sur le déplacement du pieu

$I_{p} \quad$ : facteur d'influence du talus sur la réaction latérale

$\mathrm{K}_{\mathrm{s}} \quad$ : paramètre de la courbe P-Y du PHRI (MPa.m $\left.{ }^{-1,5}\right)$

$\mathrm{M}$ : Moment de flexion à une profondeur donnée ( $\mathrm{kN} . \mathrm{m})$

$\mathrm{N}_{\mathrm{H}}$ : pente du profil de module de réaction sécant $(\mathrm{MPa} / \mathrm{m})$

P : réaction latérale du sol à une profondeur donnée $(\mathrm{kN} / \mathrm{m})$

$\mathrm{p}_{\mathrm{i}} \quad$ : pression limite de l'essai pressiométrique (MPa)

q. : résistance en pointe de l'essai au pénétromètre statique (MPa)

t : : distance du pieu à la tête du talus (m)

$\mathrm{Y}_{\mathrm{H}} \quad$ : déplacement en tête du pieu (mm)

$Y_{u} \quad$ : déplacement du pieu en surface du sol (mm)

Y : déplacement latéral du pieu à une profondeur fixée (mm)

$\mathrm{Z}$ : profondeur à partir de la surface du sol $(\mathrm{m}$ )

$\gamma_{4}$ : poids volumique sec du sable $\left(\mathrm{kN} \cdot \mathrm{m}^{-3}\right)$

$\eta$ : facteur d'homothétie de la courbe P-Y

\section{1}

\section{Introduction}

Dans les projets d'ouvrages sur pieux, il est nécessaire d'analyser le comportement des fondations sous un effort horizontal. Il s'agit d'un problème d'interaction tridimensionnelle sol-pieu assez complexe.

Actuellement, les besoins de la construction poussent à concevoir des ouvrages audacieux, implantés à proximité d'un talus, tels que les autoponts ou les constructions maritimes.

La littérature mondiale traitant de l'effet de la proximité du talus sur la résistance latérale du sol autour du pieu est assez rare. La multitude des paramètres entrant en jeu, rend difficile une approche théorique du problème et nécessite des études paramétriques expérimentales, à la lumière desquelles des méthodes pratíques de dimensionnement pourront être formulées.

On note à ce titre, l'étude présentée par Poulos, basée sur le couplage des équations de Mindlin d'une force horizontale enterrée dans un massif élastique homogène, avec celle d'une poutre infiniment longue, sous l'hypothèse d'une parfaite adhérence sol-pieu (Poulos, 1976). Gabr et Borden ont proposé un modèle de réaction latérale ultime mobilisée par un sol en pente autour d'un pieu circulaire rigide et lisse implanté en tête du talus (Borden et Gabr, 1987; Gabr et Borden, 1989), en s'inspirant du modèle de Reese pour un sol horizontal (Reese et Sullivan, 1980). Identiquement aux schémas classiques de calcul en pousséebutée basés sur l'hypothèse d'un comportement rigideplastique du sol, le modèle de Gabr et Borden ne tient pas compte du niveau des déplacements du pieu.

L'étude paramétrique expérimentale de ce problème, basée sur des essais en vraie grandeur, est une opération onéreuse et nécessitant des délais importants, du fait du grand nombre des paramètres à examiner.

Dans cet état des choses, les essais sur des modèles réduits centrifugés, de réalisation simple d'une part, et respectant les conditions de similitude d'autre part, sont les mieux placés pour l'analyse de ce problème.

La modélisation géotechnique en centrifugeuse a connu ces dernières années un essor mondial considérable. Notons que des recherches expérimentales récentes, entreprises par Terashi sur la centrifugeuse du PHRI (Ports and Harbours Research Institute) au Japon (Terashi et al., 1991), ainsi que Mezazigh sur la centrifugeuse du LCPC (Laboratoire Central des Ponts et Chaussées) en France (Mezazigh et Garnier, 1994), ont porté sur des modèles de pieux élancés $(\mathrm{D} / \mathrm{B}>10)$ et souples.

L'étude présentée ci-après, comporte l'analyse du comportement d'un pieu tubulaire court $(\mathrm{D} / \mathrm{B}=5,5)$ et rigide, implanté à proximité d'un talus sableux, ayant un angle de $26,6^{\circ}$ (soit une pente de $2 / 1$ ) et une hauteur de $3 \mathrm{~m}$, à partir des résultats d'essais sur modèles réduits, menés sur la centrifugeuse du LCPC.

\section{2}

\section{Présentation des moyens d'essais}

La centrifugeuse du LCPC, outil performant de recherche en géotechnique, est opérationnelle depuis 1986. Elle comporte un bras de $5,50 \mathrm{~m}$, et peut embarquer un modèle de 2 tonnes de masse sous une accélération centrifuge de $100 \mathrm{~g}$ (Corté et Garnier, 1986; Garnier, 1990).

Le modèle de pieu est un tube en aluminium, réduit à une échelle géométrique de $1 / 20$. Les conditions de similitude exigent que le modèle soit soumis à une accélération centrifuge de $20 \mathrm{~g}$, au cours de l'essai. Les caractéristiques du pieu prototype, noté $\mathrm{P}_{2}$, et celles de son modèle sont regroupées dans le tableau I. 


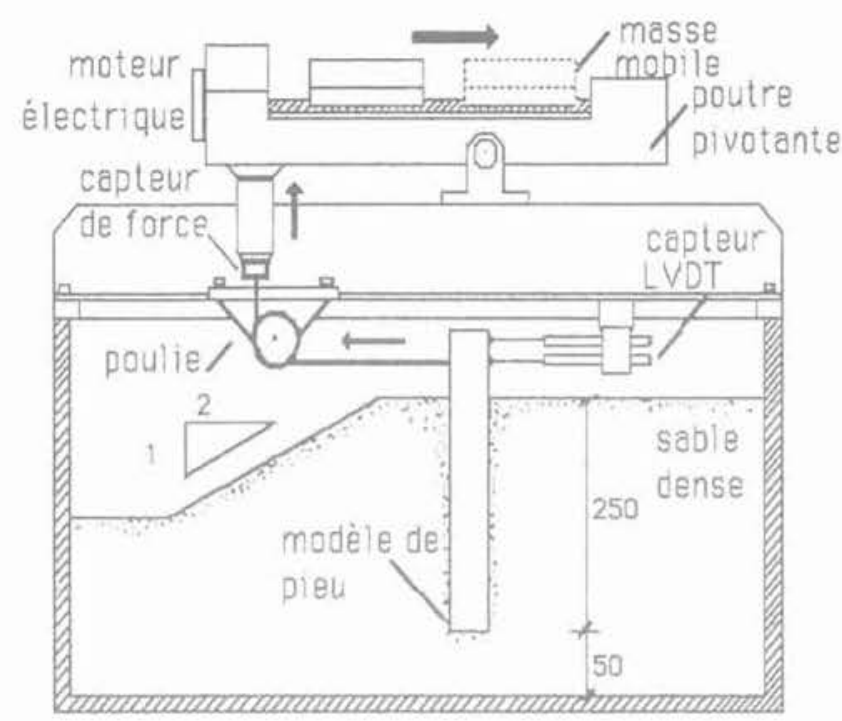

nG.1 Dispositif d'essai sur modèle centrifugé. View of testing arrangement.

TABLEAU Caractéristiques du modèle et du pieu prototype.

\begin{tabular}{l|c|c} 
& Modele & Prototype \\
\hline Échelle & $1 / 20$ & \\
$\mathrm{~B}(\mathrm{~mm})$ & 45 & $1 / 1$ \\
$\mathrm{D}(\mathrm{mm})$ & 250 & 900 \\
$\mathrm{E}_{\mathrm{i}}\left(\mathrm{N} . \mathrm{m}^{2}\right)$ & 4631 & 5000 \\
$\mathrm{e}(\mathrm{mm})$ & 50 & $741.10^{6}$ \\
\hline
\end{tabular}

Trois modèles identiques ont été utilisés, dont l'un est instrumenté par 12 paires de jauges de déformation, montées en demi-pont, et régulièrement espacées le long du pieu. La surface des tubes est rendue rugueuse, par collage du sable d'essai. Les modèles sont installés dans un conteneur, et le massif de sable est construit autour, par pluvation dans l'air.

Le chargement horizontal est assuré par un dispositif de chargement, conçu au LCPC et illustré sur la figure 1 .

Le sable d'essai, provenant du site du Rheu (Rennes), est propre et mal gradué $\left(C_{1}=2, d_{10}=\right.$ $0,3 \mathrm{~mm}$ ). Selon le système USCS (Unified Soil Classification System), ce sable appartient à la classe SP. Le massif construit est sec et très dense $\left(\gamma_{\mathrm{d}}=16,5 \mathrm{kN} / \mathrm{m}^{3}\right.$, $\mathrm{ID}=95 \%$ ). La construction du talus se fait après le remplissage du conteneur par arasage progressif. La caractérisation du massif de sable en centrifugeuse est effectuée par le contrôle de la densité, et par des essais de pénétration statique à l'aide d'un pénétromètre miniature embarqué en centrifugeuse, permettant, outre la mesure de la résistance en pointe, d'apprécier l'homogénéité du massif.

Les modèles du pieu sont placés à différentes distances de la tête du talus dont les valeurs sont regroupées dans le tableau II. Avant la réalisation du talus, un modèle a été chargé dans le massif horizontal, correspondant ainsi à une distance infinie entre le pieu et la crête du talus. Dans ce qui suit, les résultats présentés correspondent aux grandeurs prototypes du pieu simulé.
TABLEA II Distances relatives t/B.

\begin{tabular}{l|lllllll}
\hline$t / B$ & $0,44^{*}$ & $2,1^{*}$ & 3,1 & 6.55 & 9,3 & 12,6 & $\infty^{*}$ \\
\hline
\end{tabular}

$*$ : modèle instrumenté par des jauges de déformation

\section{3}

\section{Comparaison des résultats d'essais sur modèles}

En 1986, King et Fulthorpe ont réalisé, sur la centrifugeuse de Liverpool, une série d'essais sur des modèles d'un pieu rigide placé dans un massif de sable sec et dense compacté en couches de 30 à $40 \mathrm{~mm}$ d'épaisseur, par un vibreur manuel (King et Fulthorpe, 1986). L'essai sur le modèle de pieu implanté dans un sol horizontal $(\mathrm{t} / \mathrm{B}=\infty)$ et celui de King et Fulthorpe simulent en fait, le même pieu prototype, à des échelles de réduction géométrique différentes. Selon la figure 2 , les courbes de chargement sont en bonne concordance, L'écart léger, qui existe entre les déplacements mesurés au cours des deux expériences, peut être dô aux différences de caractéristiques géométriques des modèles, et au mode de construction du massif de sable. Une telle concordance confirme la bonne qualité des résultats expérimentaux présentés ici.

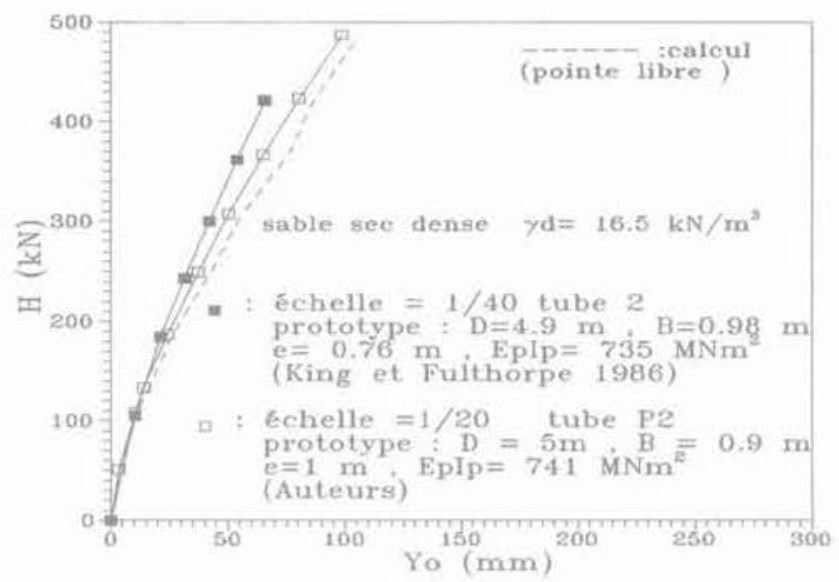

FG. 2 Comparaison des essais sur modèles. Comparison between the model tests.

\section{Effet du talus sur les moments de flexion}

Comme le montre la figure 3 , le moment de flexion, pour un effort donné, dépend peu de la présence du talus, notamment dans la moitié supérieure du pieu. La cote du moment maximal, par rapport au cas du sol horizontal, n'a pas changé.

L'effet du talus sur le moment maximal peut être défini par un facteur d'influence tel que:

$$
\operatorname{IMmax}(\mathrm{t} / \mathrm{B})=\frac{\operatorname{Mmax}(\mathrm{t} / \mathrm{B})}{\operatorname{Mmax}(\infty)}
$$




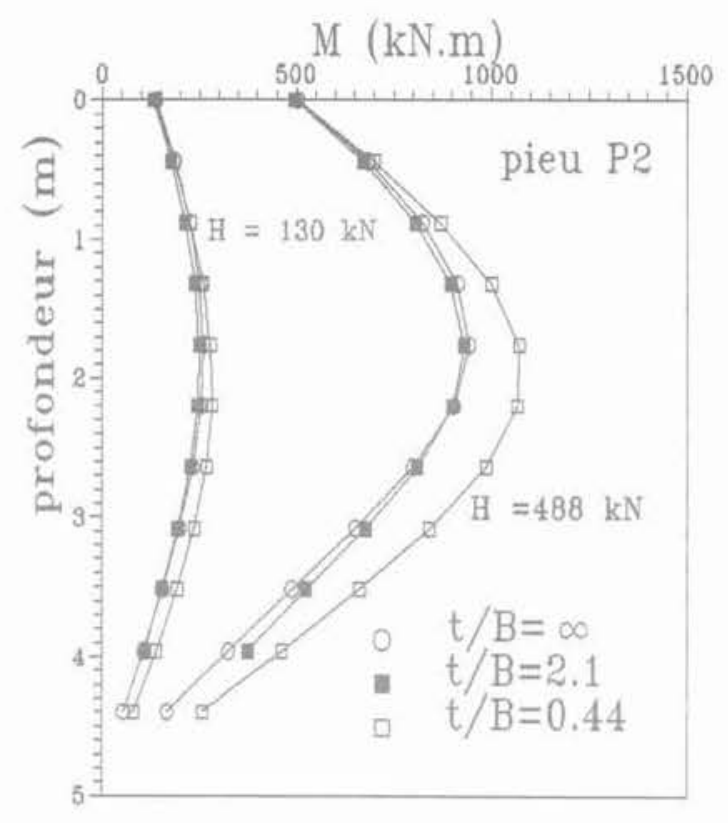

FG. 3 Effet du talus sur les moments de flexion.

The slope effect on the bending moments.

Selon le tableau III, au-delà d'une distance de 2 diamètres, le moment maximal n'est pas influencé par la présence du talus. En tête du talus ( $/ \mathrm{B}=0,44)$, l'augmentation n'est que de $10 \%$ environ.

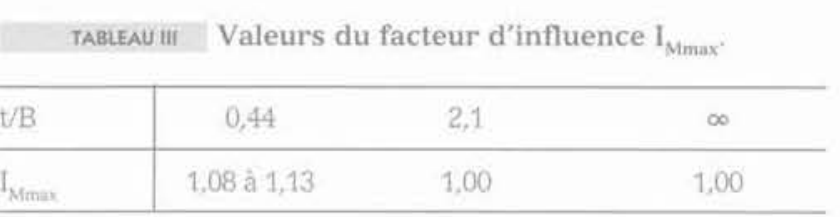

\section{5}

\section{Effet du talus sur les déplacements en tête du pieu}

La figure 4 illustre concrètement l'ampleur de l'effet du talus. Par rapport à un sol horizontal, les déplacements en tête du pieu, pour $t / B=0,44$, sous l'effort maximal en tête ont triplé.

L'effet du talus sur les déplacements en tête peut être défini par un facteur d'influence $\mathrm{I}_{\mathrm{\gamma H}}$ tel que :

$$
I_{Y A}(t / B)=\frac{Y_{H}(t / B)}{Y_{H}(\infty)}
$$

D'après la figure 5, au-delà d'une distance de 6,5 diamètres, la proximité du talus cesse d'avoir un effet sur les déplacements en tête du pieu.

\section{6}

\section{Analyse des courbes P-y}

La courbe de réaction horizontale, notée universellement P-Y, décrit la loi de comportement de l'interface sol-pieu, généralement non linéaire.

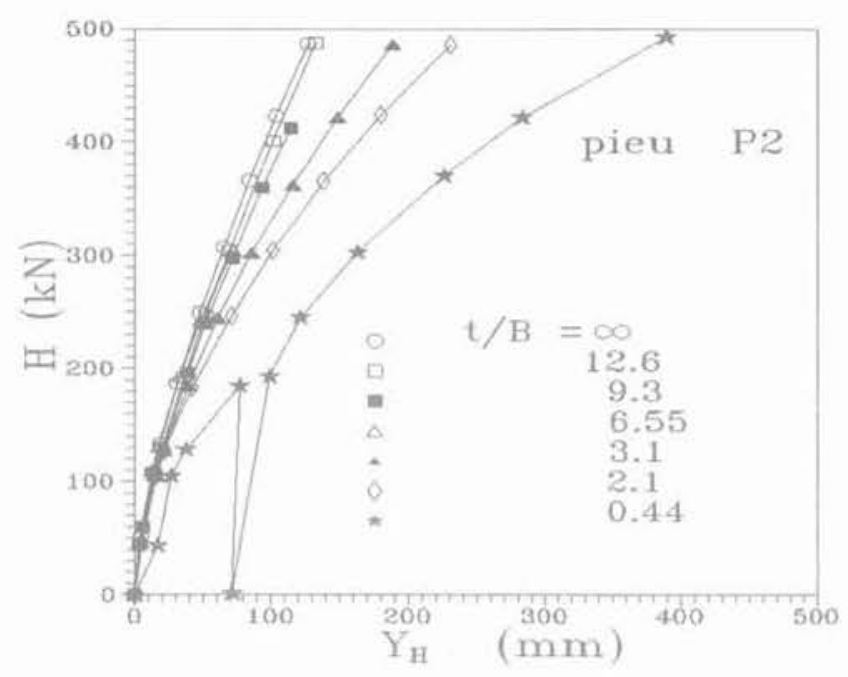

FIG.4 Effet du talus sur les déplacements en tête.

The slope effect on the top deflections.

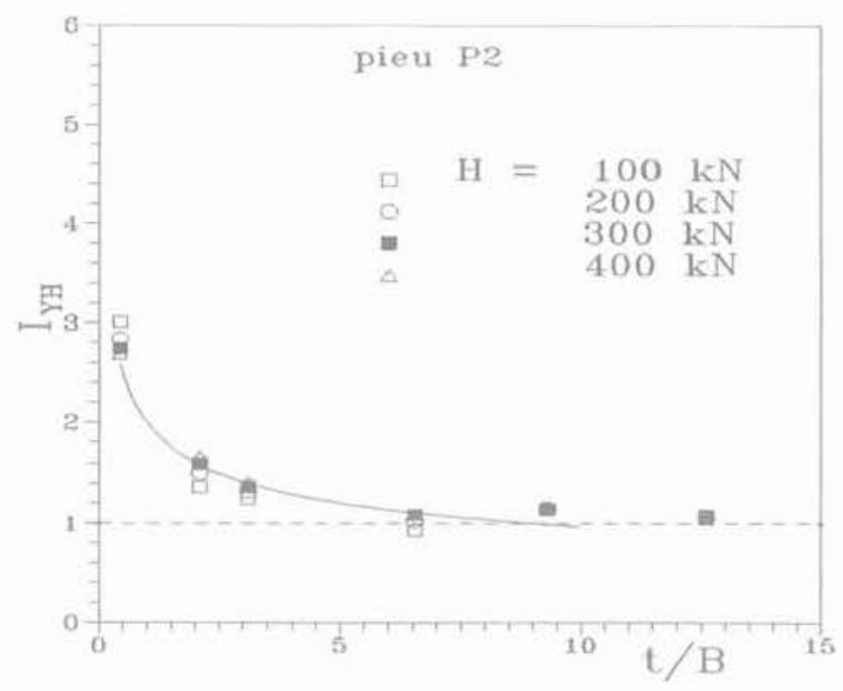

FIG.5 Facteur d'influence sur les déplacements en tête.

The influence factor on the top deflections.

Ces dernières années, les méthodes de calcul des pieux, sur la base de la théorie du module de réaction, ont connu un développement important, ce qui a accéléré les recherches expérimentales sur les courbes de réaction

La réaction latérale du sol, le long du pieu, est obtenue, au signe près, par double dérivation de la courbe des moments :

$$
P=-\frac{d^{2} M}{d Z^{2}}
$$

Le déplacement latéral est obtenu par double intégration de la courbe des moments:

$$
Y=\frac{1}{E_{p} \cdot I_{p}} \cdot \iint(M \cdot d Z) \cdot d Z
$$



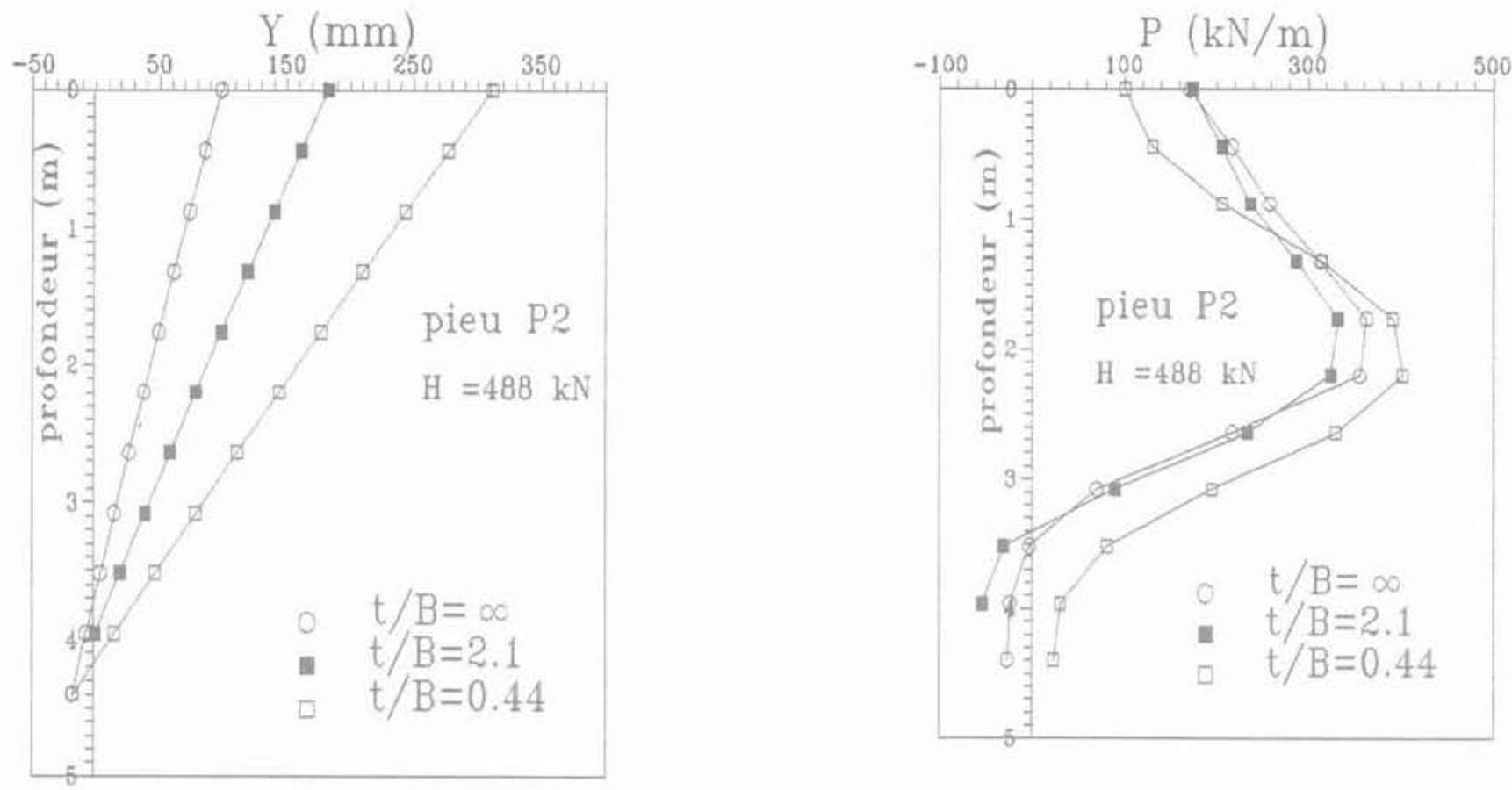

FG.6 Effet du talus sur les profils de déplacement et de réaction latérale.

The slope effect on theprofiles of displacement and lateral reaction of soil.

La courbe des moments est ajustée par des fonctions splines quintiques paramétrées par un coefficient d'ajustement définissant le degré de fidélité de la courbe de lissage aux points à lisser. Le critère de choix de la courbe de lissage est la vérification des équations de la statique le long du pieu, à partir du profil de réaction latérale, et les efforts mesurés en tête, à $10 \%$ près (Bouafia, 1990; Bouafia et Garnier, 1991a; Bouafia, 1994). Les courbes expérimentales du moment de flexion ont été introduites dans le logiciel de lissage SLIVALIC-5 du LCPC.

La figure 6 illustre, à titre d'exemple, les profils de réaction latérale du sol, et des déplacements du pieu, pour un effort en tête de $488 \mathrm{kN}$.

Le profil de réaction latérale est caractérisé par une faible mobilisation de la contre-butée près de la pointe.
Sur la même figure, le profil de déplacement du pieu est pratiquement linéaire, typiquement d'un pieu rigide répondant au chargement horizontal par un déplacement, sa flexibilité étant relativement négligeable. La figure 6 montre, en outre, que la proximité du talus a pour effet de modifier les déplacements et la réaction du sol, tout le long du pieu.

A une profondeur donnée, le coefficient d'influence sur la réaction latérale peut être défini par:

$$
I_{p}(t / B)=\frac{P(t / B)}{P(\infty)}
$$

Un exemple de profil de ce facteur est celui illustré par la figure 7, correspondant au cas $t / B=0,44$. Il dépend
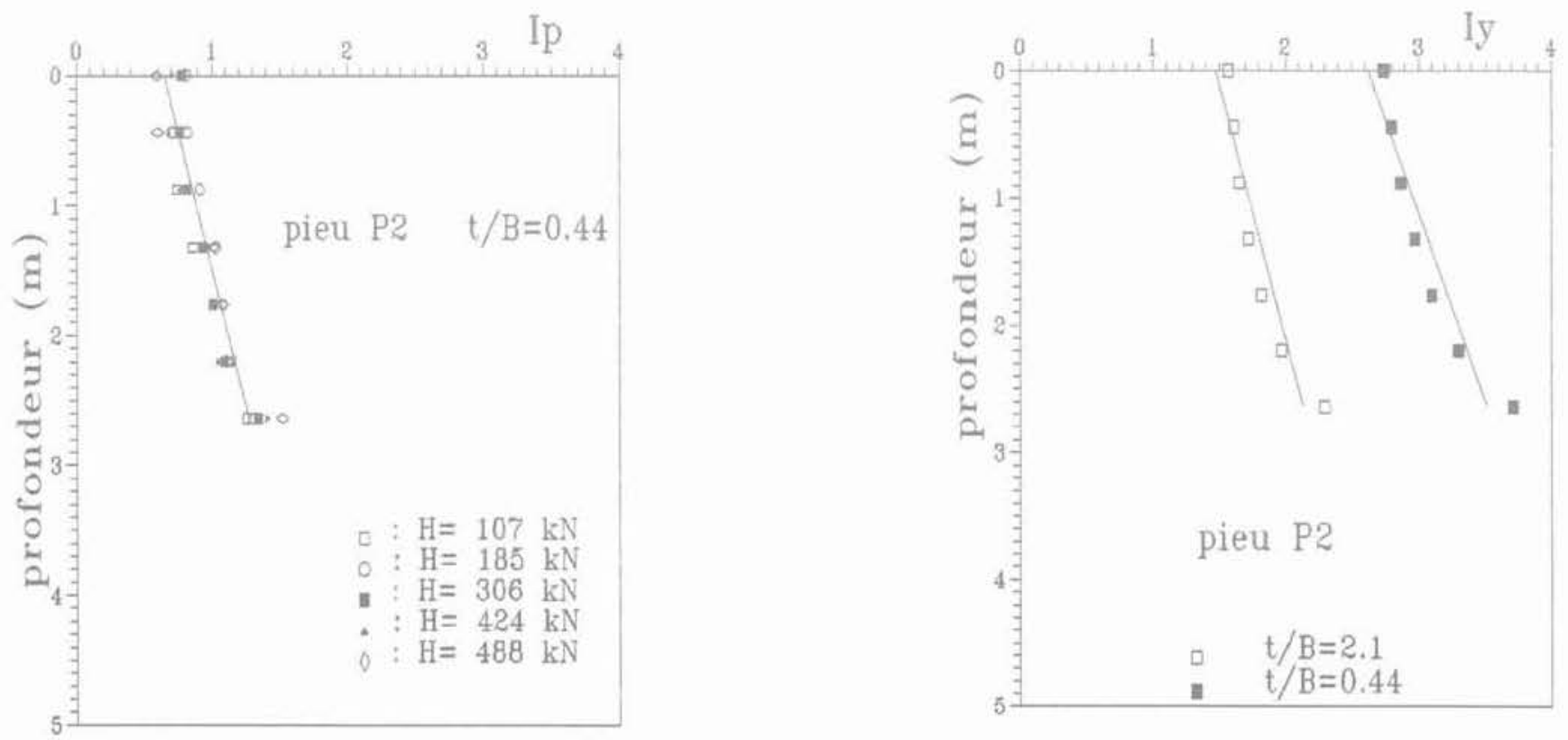

FG: 7 Facteurs d'influence du talus sur la réaction latérale et les déplacements.

The influence factor on the lateral reaction and displacements. 
peu du niveau de chargement horizontal, et varie pratiquement d'une façon linéaire avec la profondeur.

Le facteur d'influence du talus sur les déplacements latéraux du pieu est défini par

$$
I_{Y}(t / B)=\frac{Y(t / B)}{Y(\infty)}
$$

Les profils de $I_{\psi}$, sur la même figure, correspondant à des valeurs moyennes de ce facteur pour différents niveaux de chargement, et ont une allure pratiquement linéaire, du moins sur la première moitié du pieu. A proximité du centre de rotation, les déplacements étant faibles, les valeurs du facteur d'influence deviennent ainsi imprécises.

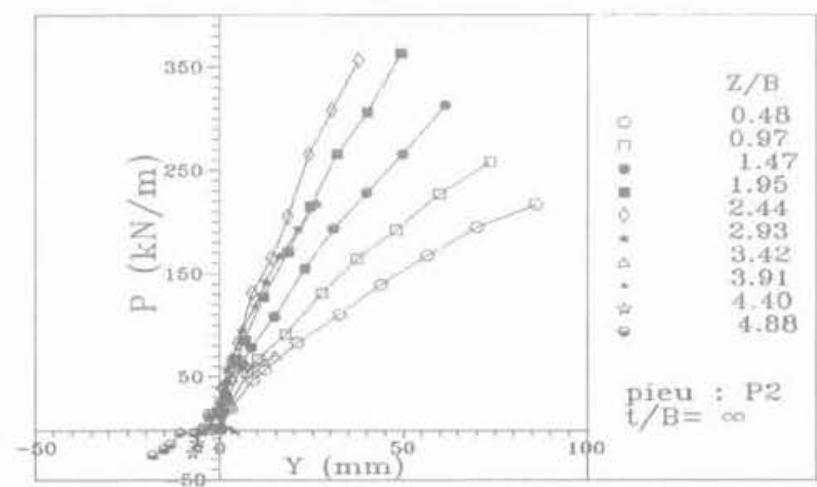

Fig.8 Courbes $\mathrm{P}-\mathrm{Y}$ du pieu $\mathrm{P}_{2}$ dans un sol horizontal.

$\mathrm{P}-\mathrm{Y}$ curves of pile $\mathrm{P}_{2}$ in a horizontal soil.

Sur les figures 8 et 9 , sont illustrées les courbes P-Y correspondant à des distances relatives $\mathrm{t} / \mathrm{B}$ de 0,44 et $\infty$,

Les courbes sont non linéaires, et les sections superficielles du pieu, subissant des grands déplacements, sont caracterisées par des paliers horizontaux apparaissant sur les courbes P-Y.

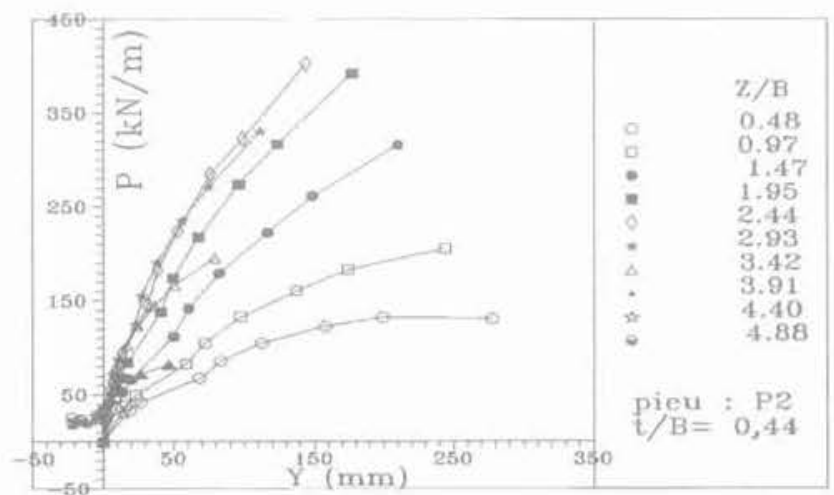

FIG.9 Courbes $\mathrm{P}-\mathrm{Y}$ du pieu $\mathrm{P}_{2}$ pour $\mathrm{t} / \mathrm{B}=0,44$, $P$ - $Y$ curves of pile $P_{2}$ for $t / B=0,44$.

Un calcul à rebours du pieu, à partir des courbes P$\mathrm{Y}$ ainsi construites, a été mené à l'aide du logiciel PILATE, mis au point au LCPC, pour le calcul des pieux chargés horizontalement par la théorie du module de réaction.

Selon la figure 10, les déplacements prévus du pieu $\mathrm{P}_{2}$, à différentes distances de la tête du talus coíncident parfaitement avec ceux mesurés. A titre d'exemple, la figure 11 illustre la comparaison des moments de flexion mesurés et ceux calculés dans le cas du sol horizontal, avec une bonne concordance (Bouguerra, 1991).

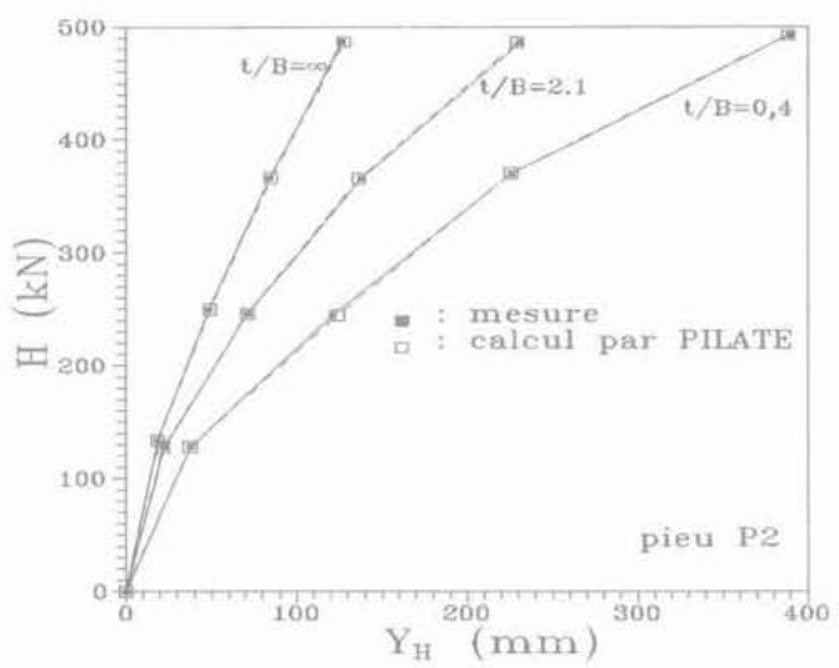

FG. 10 Comparaison des déplacements mesurés et calculés.

Comparison between measured and predicted displacements.

Ce calcul permet ainsi, d'une part de valider la méthode de construction des courbes P-Y et, d'autre part, de montrer une bonne homogénéité entre les différentes mesures effectuées sur le modèle.

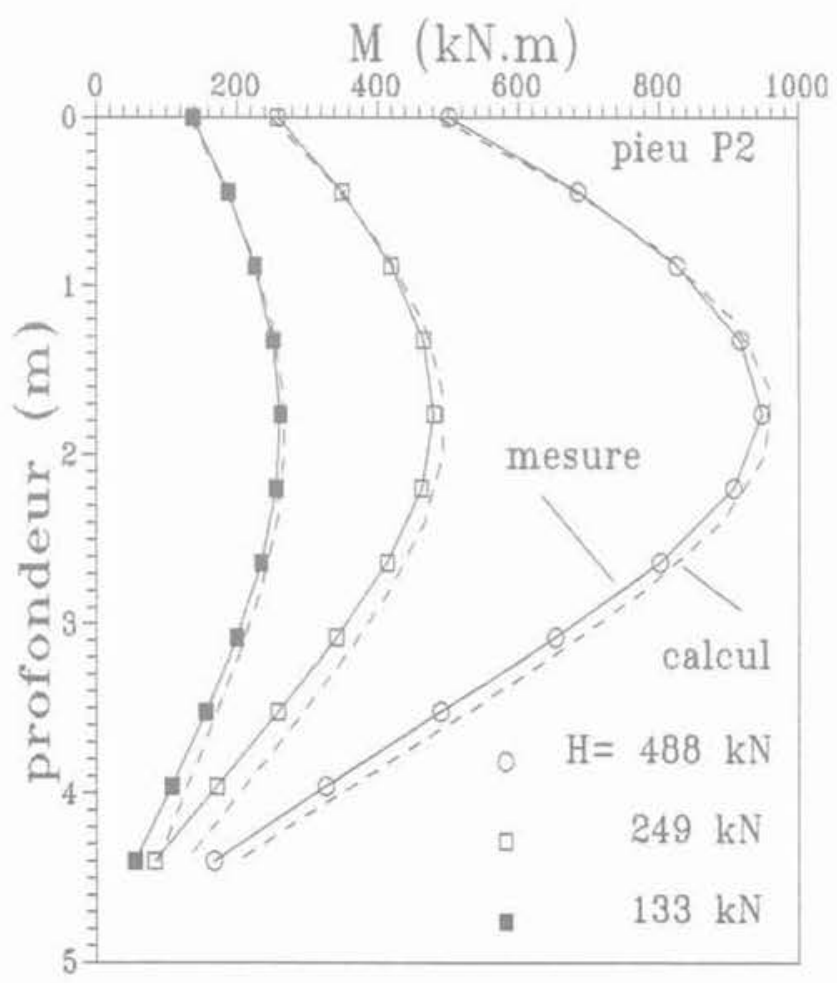

घG. 11 Comparaison des moments mesurés et calculés.

Comparison between measured and computed bending moments. 


\section{Analyse du module de réaction latérale}

Le module de réaction sécant :

$$
E_{S}=\frac{P}{Y}
$$

est une grandeur décrivant le comportement non linéaire de l'interface sol-pieu. D'après la figure 12 , pour des efforts en tête de $130 \mathrm{kN}$ et de $488 \mathrm{kN}$, les profils de ce module sont pratiquement linéaires avec la profondeur, ce qui confirme les recommandations de la littérature du pieu chargé latéralement dans un massif sableux (Bouafia, 1990; Bouafia et Garnier, 1991b; Bouafia et Bouguerra, 1995).

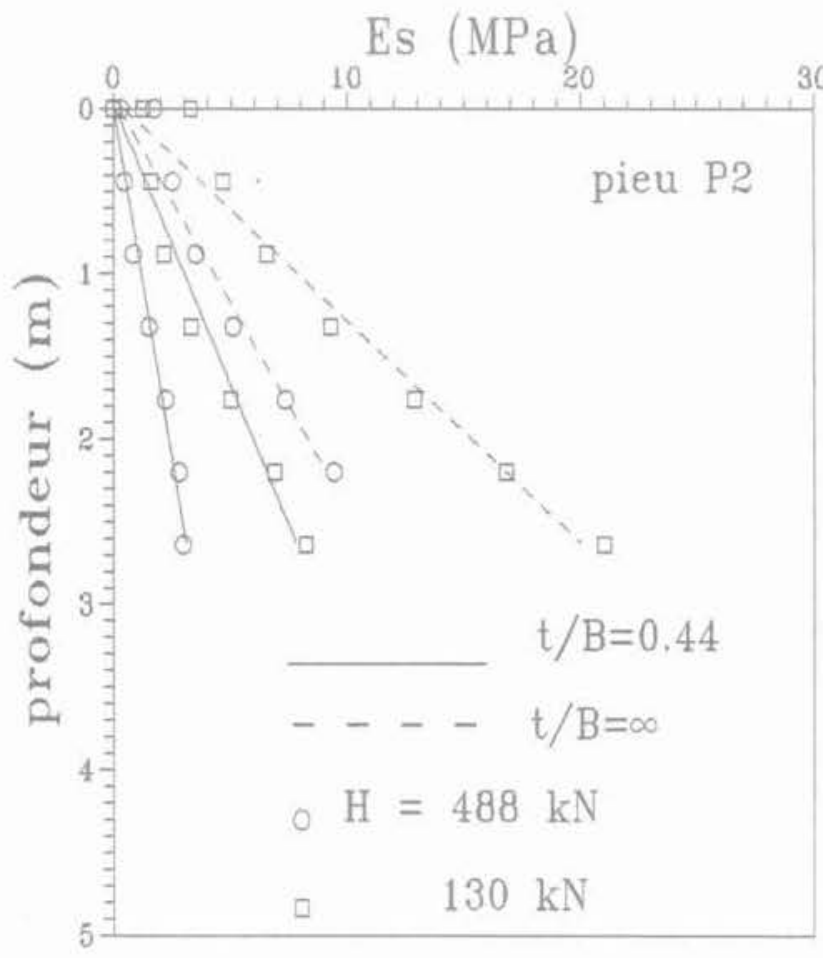

FIG, 12 Profils du module de réaction sécant. Profiles of secant subgrade reaction modulus.

Il est intéressant de constater que la présence du talus n'a pas modifié l'allure linéaire du profil de module de réaction. Au-delà d'une profondeur de $4 \mathrm{~B}$, et quelle que soit la position du pieu par rapport au talus, les déplacements ainsi que la réaction latérale du sol deviennent très faibles. Les valeurs du module de rêaction seront, par conséquent, grossières. Dans ce qui suit, on limite l'analyse en deçà de cette profondeur.

En considérant un pieu court et rigide, ayant un profil de déplacements linéaires avec la profondeur, une pointe libre, et chargé horizontalement dans un massif ayant un module de réaction de la forme :

$$
E_{\mathrm{S}}=\mathrm{N}_{\mathrm{H}} \cdot \mathrm{Z}
$$

le déplacement en surface peut être calculé, à partir de l'équilibre statique du pieu, comme suit :

$$
\mathrm{Y}_{\mathrm{O}}=18 \frac{\mathrm{H}}{\mathrm{N}_{\mathrm{H}} \cdot \mathrm{D}^{2}}+24 \frac{\mathrm{H} \cdot \mathrm{e}}{\mathrm{N}_{\mathrm{H}} \cdot \mathrm{D}^{3}}
$$

L'ajustement des profils du module de réaction par des droites passant par l'origine a permis de calculer les déplacements en surface par l'expression précédente. La comparaison avec les déplacements mesurés sur la figure 2 montre une très bonne concordance. Le module sécant, issu des courbes P-Y, permet ainsi de décrire correctement le comportement du pieu.

Le module tangent à l'origine de la courbe, représentant la raideur horizontale initiale du sol, a été obtenu en effectuant une interpolation quadratique du tronçon initial de la courbe P-Y (Bouafia, 1990 ; Bouafia et Garnier, 1991b), et illustré sur la figure 13. Ces profils ont une allure sensiblement linéaire et se prêtent bien à un ajustement par une droite passant par l'origine. Les valeurs de la pente du profil de module de réaction tangent, pour différentes positions du pieu par rapport à la crête du talus, sont regroupées dans le tableau IV.

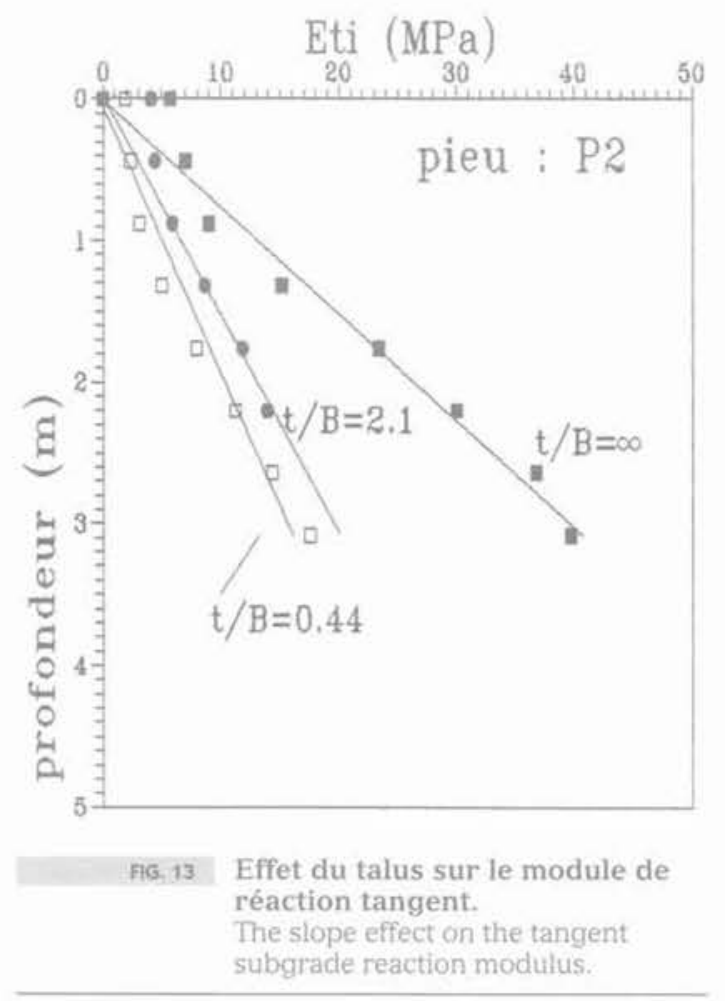

TABLEAUN Valeurs de a et de $\mathrm{I}_{\mathrm{E}}$.

\begin{tabular}{l|c|cc}
$\mathrm{t} / \mathrm{B}$ & 0,44 & 2,10 & $\mathrm{e}$ \\
\hline $\mathrm{a}(\mathrm{MPa} / \mathrm{m})$ & 5,40 & 6,50 & 13,30 \\
\hline $\mathrm{I}_{\mathrm{E}}$ & 0,40 & 0,50 & 1,00 \\
\hline
\end{tabular}

L'influence de la proximité du talus sur le module de réaction initial, peut être définie par le facteur:

$$
I_{E}(t / B)=\frac{E_{t i}(t / B)}{E_{t I}(\infty)}
$$

Les valeurs du tableau IV, montrent qu'en tête du talus, le module tangent diminue environ de la moitié par rapport au cas du sol horizontal.

Il est remarquable, d'après l'allure linéaire des profils illustrés sur la figure 13, que dans le cas d'un pieu court et rigide, l'influence de la proximité du talus sur le 
module de réaction initial est la même pour toutes les profondeurs,

Les valeurs obtenues du module tangent du sol horizontal ont été comparées aux recommandations de la théorie pressiométrique de Ménard (Baguelin et al. 1978), en se basant sur la corrélation classique pour le sable (Van Wambecke et d'Hemricourt, 1982):

$$
\frac{E_{M}}{q_{o}}=1 \text { à } 1,5
$$

La résistance en pointe a été mesurée par un minipénétromètre statique de $12 \mathrm{~mm}$ de diamètre, au cours de la centrifugation du modèle.

Selon les recommandations de 1'Institut japonais des recherches portuaires PHRI, la loi de réaction latérale est formulée comme suit (Kubo, 1965; PHRI, 1980) :

$$
\mathrm{P}=\mathrm{K}_{\mathrm{S}} \cdot \mathrm{B} \cdot \mathrm{Z} \cdot \mathrm{Y}^{1 / 2}
$$

En se basant sur cette expression, Terashi a proposé des valeurs au paramètre $\mathrm{K}_{\text {c }}$ en fonction du diamètre du pieu, à partir des résultats d'essais sur modèles centrifugés dans le sable (Terashi et al., 1989). Comme pour les essais présentés ici, le massif de sol a été construit par pluviation du sable dans l'air. Un bon accord est visible sur la figure 14 entre le module de réaction tangent expérimental et celui recommandé par Ménard. On peut constater une certaine coincidence avec le module de réaction recommandé par Terashi, notamment pour les faibles profondeurs.

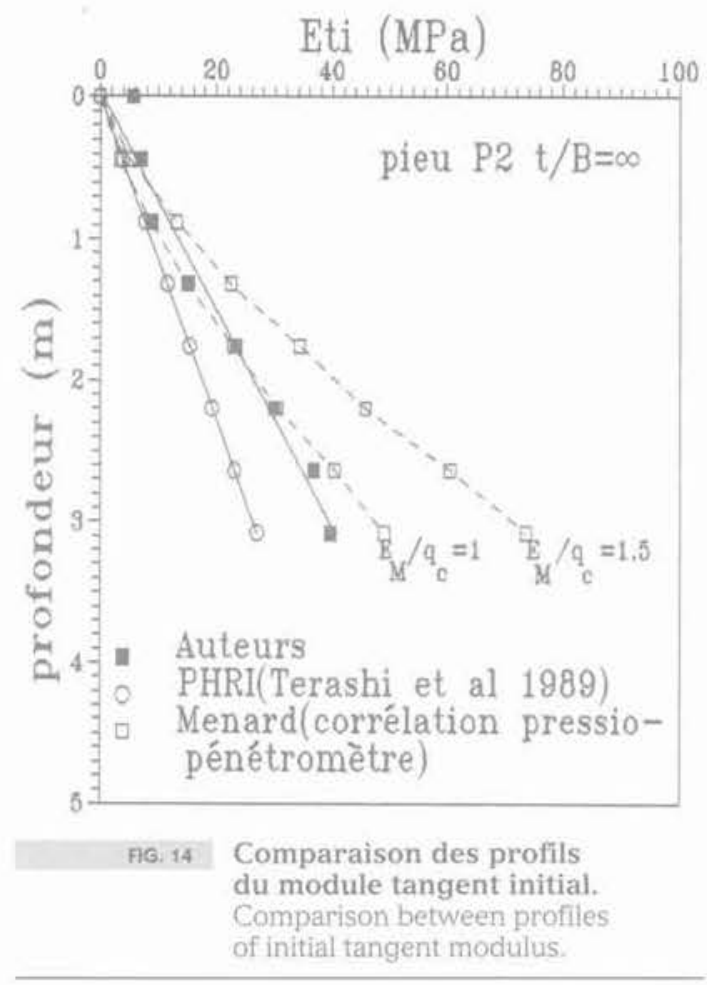

8

\section{Modifications de la courbe P-y par l'effet du talus}

Dans ce qui suit, on montre qu'il est possible de prévoir correctement le comportement du pieu près d'une pente, en effectuant une modification des courbes P-Y du même pieu dans un sol horizontal. Les paramètres de modification dépendent évidemment des caractéristiques du système pieu/sol. De ce fait, il est précoce de recommander, dans cette étude, des valeurs à ces paramètres. Seule l'analyse d'autres géométries du système sol/pieu peut confirmer une telle méthode d'analyse.

Deux procédures simplifiées de correction sont proposées.

- Procédure 1: correction de la courbe P-Y par affinité

On se propose de construire la courbe P-Y du pieu près du talus, en effectuant une affinité de l'axe des déplacements par le facteur $I_{y}$, et celui de la réaction du sol par I (Fig. 15). Ces facteurs dépendent évidemment de $\mathrm{t} B \mathrm{~B}$ et de la profondeur. On suppose que :

- en deçà du pied du talus, $I_{y}$ et $I_{p}$ augmentent línéairement avec la distance $t$ :

- au-delà du pied du talus, la courbe P-Y du sol horizontal est conservée, quelle que soit la distance t:

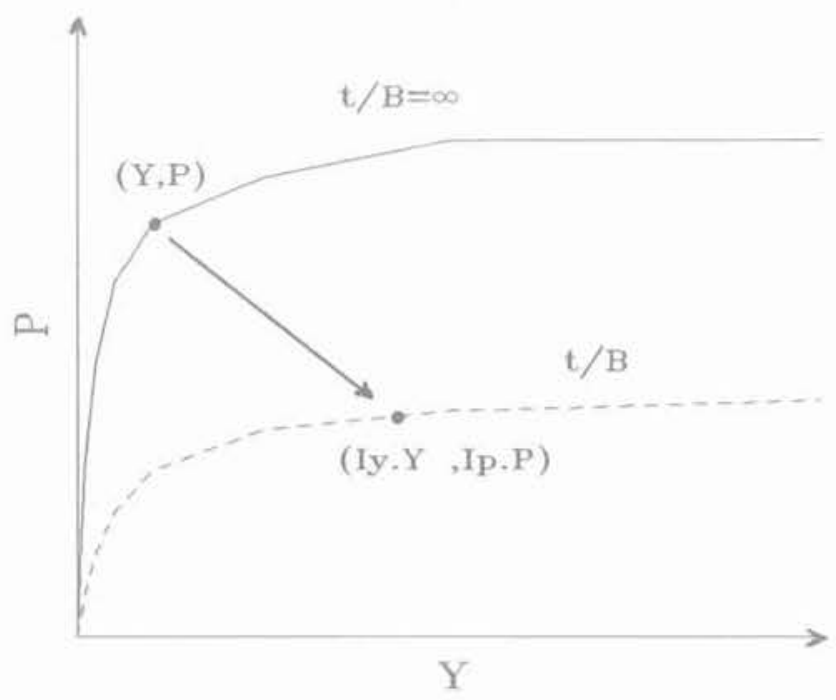

FiG. 13 Modification de la courbe P-Y par affinité. Modification of P-Y curve by affinity.

- Procédure 2 : correction de la courbe P-Y par homothétie

On s'intéresse à un facteur d'homothétie n, pour un déplacement horizontal donné, tel que :

$$
\eta=\frac{P(Y, Z, t / B)}{P(Y, Z, \infty)}
$$

Il est évident que ce facteur est limité pour les petits déplacements par $I_{E}$, et pour les grands déplacements par $I_{p}$ :

$$
\mathrm{I}_{\mathrm{E}}<\eta<\mathrm{I}_{\mathrm{P}}
$$

Ainsi, comme le montre la figure 16, la courbe P-Y du sol horizontal sera corrigée pour les petits déplacements en diminuant le module tangent initial par $\mathrm{I}_{\mathrm{E}}$, et pour les grands déplacements en multipliant la réaction ultime du sol par $I_{p}$.

On suppose que :

- en-deçà du pied du talus, $I_{E}$ et $I_{p}$ augmentent linéairement avec la distance $t$;

- au-delà du pied du talus, la courbe P-Y du sol horizontal est conservée, quelle que soit la distance $t$. 


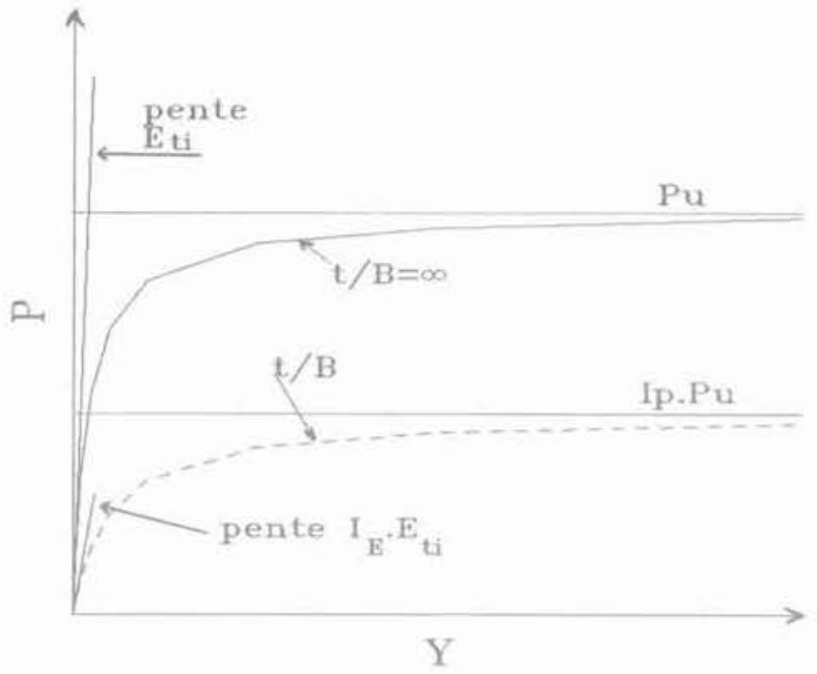

FiG. 16 Modification de la courbe P-Y par homothétie. Modification of P-Y curbe by homothery
9

\section{Prévision du comportement du pieu à proximité du talus}

Les procédures proposêes sont à valider, en effectuant une prévision du comportement du pieu $\mathrm{P}_{2}$ implanté à 3,1 diamètres de la tête du talus, l'outil de calcul étant le logiciel PILATE. Pour chaque procédure, la courbe $P-Y$ du pieu $P_{2}$ dans un sol horizontal est corrigée et introduite dans le code de calcul.

\section{- Procédure 1}

Sur la figure 17, on remarque une bonne concordance entre les déplacements mesurés et ceux calculés à partir des courbes $\mathrm{P}-\mathrm{Y}$ expérimentales ainsi corrigées.

- Procédure 2

Cette procédure est appliquée à la courbe P-Y proposée par Ménard. Cette demière est trilinéaire, formée initialement d'une droite dont la pente est proportionnelle au module pressiométrique, Pour les grands déplacements, la courbe comporte un palier de réaction latérale ultime égale à la pression limite p, mesurée au pressiomètre, multipliée par le diamètre B du pieu. En rentrant la corrélation suivante (Van Wambecke et d'Hemricourt, 1982):

$$
\frac{q_{c}}{p_{1}}=7
$$

les courbes P-Y pressiométriques peuvent être construites.

Au préalable, un calcul du pieu dans un sol horizontal (Fig. 17) a été effectué à partir de ces courbes. Comme le montre la figure 17 , les courbes P-Y pressiométriques ne permettent pas de prévoir correctement les grands déplacements du pieu. Cette constatation rejoint celles d'autres auteurs ayant effectué un calcul similaire, confronté aux résultats d'essais sur des pieux en vraie grandeur (Baguelin et Jézéquel, 1972 ; Frank, 1984; Baguelin et al., 1990).

Par la suite, la courbe P-Y a été corrigée et introduite dans PIL.ATE. Selon la figure 17, la comparaison des déplacements mesurés et prévus montre une bonne concordance pour les faibles niveaux de chargement.

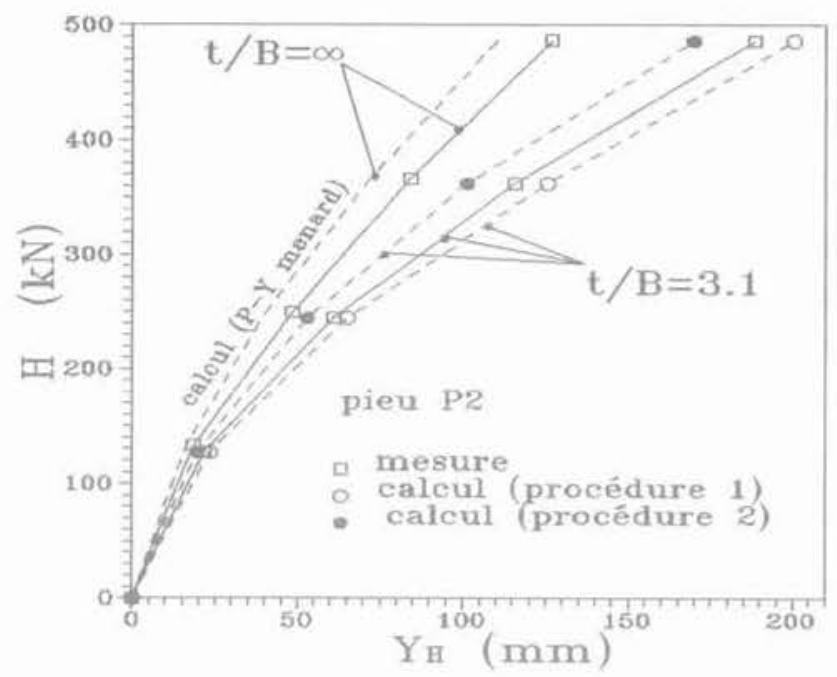

\#G,17 Comparaison des déplacements mesurés et calculés.

Comparison between measured and predicted displacements.

Le calcul prévisionnel montre ainsi qu'il est possible d'introduire des paramètres de transformation géométrique de la courbe P-Y du sol horizontal indépendants du niveau de déplacement du pieu, et dont la loi de variation dans l'espace est simple (variations linéaires en profondeur et avec $\mathrm{t} / \mathrm{B}$ ).

\section{0}

\section{Conclusion}

La modélisation en centrifugeuse du comportement d'un pieu court et rigide, instrumenté par des jauges de déformation a permis d'étudier l'effet de la proximité d'un talus sableux dense de $3 \mathrm{~m}$ de hauteur et d'une pente de $2 / 1$ sur les déplacements et le moment fléchissant du pieu.

La distance limite, au-delà de laquelle les déplacements en tête ne sont plus influencés par la présence du talus est de 6,5 diamètres, pour le système sol-pieu étudié.

Le moment fléchissant est peu sensible à la proximité du talus, avec une augmentation au plus de $16 \%$ du moment maximal.

Il est possible de décrire correctement le comportement du pieu près d'une pente en effectuant une correction adéquate de la courbe P-Y du même pieu implanté dans un sol sans pente.

La généralisation d'une telle méthode d'étude de l'effet du talus à partir des courbes P-Y nécessitera le recours à des études paramétriques expérimentales, afin de conclure sur sa validité.

\section{Remerciements}

Les auteurs sont reconnaissants à l'équipe de la section Mécanique des sols et Centrifugeuse du LCPC, centre de Nantes, pour leur précieuse aide au cours de la préparation et l'exécution des essais en centrifugeuse. 
Baguelin F, Jézéquel J.F. - Étude expérimentale du comportement des pieux sollicités latéralement. Annales de I'TTBTP, $n^{\circ} 297$, 1972, p.155-201.

Baguelin F. et al. - The pressuremeter and foundation engineering. Series on rock ans soil mechanics. Transtech Publications, 1978 .

Baquelin F. et al. - The Menard pressuremeter test and the behaviour of laterally loaded piles in sands. $3^{\text {ru }}$ International Symposium on Pressuremeters, Oxford, 2-6 April 1990

Borden R.H., Gabr M.A. - ITBASE : Lateral pier analysis including base and slope effect. Research report $n^{\circ}$ FHWA/NC/86-001, Centre for Transportation Engineering Stuclies, North Carolina, 1987.

Bouafia A. - Modélisation des pleux chargés latéralement en centrifugeuse. Thèse de doctorat en génie civil soutenue le 20 décembre 1990, á l'École nationale supérieure de mécanique de Nantes, France, 1990.

Bouafia A.. Garnier J. - Comportement d'un pieu isolé chargé latẻralement dans le sable. Colloque international Fondations profondes, Paris, 19-21 mars 1991. $1991 \mathrm{a}$.

Bouafia A., Garnier J. - Experimental study of P.Y curves for piles in sand. Proceedings of the $2^{\text {nd }}$ international Conference Centrifuge 91. Boulder Colorado, 13-14 June 1991, 1991b, p. 261-268.

Bouafia A. - Etude expérimentale du chargement latéral cyclique répétẻ des pieux isolés dans le sable en centrifugeuse.
Revue Canadienne de Géotechnique, vol. 31. $n^{\circ} 5,1994$, p. $740-748$.

Bouafia A., Bouguerra A. - Modélísation en centrifugeuse du comportement d'un pieu flexible chargé horizontalement à proximité d'un talus, Revue Canadienne de Géotechnique, vol. 32, avril 1995.

Bouguerra A. - Effet de la proximité d'une pente sur la résistance latérale d'un pieu. Mémoire du Diplôme d'études approfondies, soutenu le 12 juillet 1991 à l'ENSM de Nantes, France, 1991.

Corté J.-F., Garnier J. I Une centrifugeuse pour la recherche en géotechnique. Builetin de Liaison des laboratoires des ponts et chaussées, $n^{\circ} 146$, nov-déc 1986 .

Frank R. - Etudes théoriques des fondations profondes et d'essais en place par autoforage dans les LPC et résultats pratiques. Rapport de recherche du LCPC $n^{\circ} 128$, juin 1984.

Gabr M. Borden R.H. - Lateral response of piers in sloping soil profiles. Proceedings of the $12^{\text {th }}$ international conference on soil mechanics and foundation engineering. Rio de Janeiro, vol. 2, 1989, p. 1197-1199.

Garnier J. - La centrifugeuse du LCPC : un nouveau moven d'essais en macrogravité. Colloque international ASTELAB 90, Paris, $11-15$ juin 1990 , p. 417-422.

King C., Fulthorpe J. - Centrifuge model tests on laterally loaded single piles. Proceedings of the $111^{\mathrm{rt}}$ Indian Conference on Ocean Engineering. ITT Bombay, 1986

Kubo K. - Experimental study of the behaviour of laterally loaded piles. Procee- dings of the $6^{\text {th }}$ International Conference on Soil Mechanics and Foundation Engineering, Montreal, vol. 2, 1965 . p. $275-279$.

Mezazigh S., Garnier J. - Laterally loaded piles: effect of the slope and density of the soil mass on the $P-\gamma$ reaction curves. Proceedings of the $3^{\text {ro }}$ international Conference Centrifuge 94. Singapore. 1994.

PHRI - Technical standards for ports and harbours facilities in Japan, 1980, p. 128 . 133.

Poulos H.G. - Behaviour of laterally loaded piles near cut or slope, Australian Geomechanics Journal, vol. G6, $n^{\circ} 1,1976$.

Reese L.C., Sullivan W.R. - Documentation of Computer program COM-624, Parts I and II. Department of Civil Engineering. the University of Texas at Austin August 1980.

Terashi M., et al. - Centrifuge modeling of a laterally loaded pile. Proceedings of the 12th international conference on soil mechanic and foundation engineering. Rio de Janeiro vol. 1. p. 991-994.

Terashi M. et al. - Lateral resistance of a long pile near the slope. Proceedings of the $2^{\text {nd }}$ international conference Centrifuge 91, Boulder, Colorado, 13-14 June 1991, p. 245-252.

Van Wambecke et d'Hemricourt - Correlation between the results of static or dynamic probing and pressuremeter tests. Proceedings of the $2^{\text {nd }}$ European Symposium on Penetration testing, Amsterdam, 1982. 